This item was submitted to Loughborough's Research Repository by the author.

Items in Figshare are protected by copyright, with all rights reserved, unless otherwise indicated.

\title{
Cross view capture for stereo image super-resolution
}

PLEASE CITE THE PUBLISHED VERSION

https://doi.org/10.1109/TMM.2021.3092571

\section{PUBLISHER}

Institute of Electrical and Electronics Engineers

\section{VERSION}

AM (Accepted Manuscript)

\section{PUBLISHER STATEMENT}

(c) 2021 IEEE. Personal use of this material is permitted. Permission from IEEE must be obtained for all other uses, in any current or future media, including reprinting/republishing this material for advertising or promotional purposes, creating new collective works, for resale or redistribution to servers or lists, or reuse of any copyrighted component of this work in other works.

\section{LICENCE}

All Rights Reserved

\section{REPOSITORY RECORD}

Zhu, Xiangyuan, Kehua Guo, Hui Fang, Liang Chen, Sheng Ren, and Bin Hu. 2021. "Cross View Capture for Stereo Image Super-resolution”. Loughborough University. https://hdl.handle.net/2134/14827764.v1. 


\title{
Cross View Capture for Stereo Image Super-Resolution
}

\author{
Xiangyuan Zhu, Kehua Guo, Member, IEEE, Hui Fang, Liang Chen, Sheng Ren and Bin Hu
}

\begin{abstract}
Stereo image super-resolution exploits additional features from cross view image pairs for high resolution (HR) image reconstruction. Recently, several new methods have been proposed to investigate cross view features along epipolar lines to enhance the visual perception of recovered HR images. Despite the impressive performance of these methods, global contextual features from cross view images are left unexplored. In this paper, we propose a cross view capture network (CVCnet) for stereo image super-resolution by using both global contextual and local features extracted from both views. Specifically, we design a cross view block to capture diverse feature embeddings from the views in stereo vision. In addition, a cascaded spatial perception module is proposed to redistribute each location in feature maps according to the weight it occupies to make the extraction of features more effective. Extensive experiments demonstrate that our proposed CVCnet outperforms the state-of-the-art image super-resolution methods to achieve the best performance for stereo image super-resolution tasks. The source code is available at https://github.com/xyzhu1/CVCnet.
\end{abstract}

Index Terms-Stereo image, super-resolution, cross view capture, spatial perception.

\section{INTRODUCTION}

$\mathbf{S}$ TEREO image super-resolution (SR) is attracting considerable attention due to its great value in 3D applications. Certainly, there has been a high demand for 3D contents with finer resolution [1]-[6] since the rise of immersive technologies, including high resolution (HR) 3DTV, augmented reality (AR), and virtual reality (VR). However, limitations on stereo imaging capture devices, e.g., the use of dual cameras on mobile phones, may produce low resolution (LR) stereo image pairs. In addition, narrow network bandwidth also restricts the transmission of high resolution stereo images. Stereo image SR aims to generate HR stereo image pairs from their low resolution counterparts to significantly enhance their visual perception. Thus, this technique has great potential in improving the user experience when deploying immersive services.

Recently, many deep learning (DL) based SR algorithms have started exploring multi-image sources to further improve SR performance since convolutional neural network (CNN) based methods are widely utilized on single image super-resolution (SISR) tasks [7]-[11]. These methods can

*Corresponding author: Kehua Guo.

Xiangyuan Zhu, Kehua Guo, Liang Chen, Sheng Ren and Bin Hu are with the School of Computer Science and Engineering, Central South University, Changsha 410083, China (e-mail: zhuxiangyuan@csu.edu.cn; guokehua@csu.edu.cn; chenliang1@csu.edu.cn; rensheng@csu.edu.cn; hubincsu@csu.edu.cn; ).

Hui Fang is with the Computer Science Department, Loughborough University, Loughborough, U.K. LE11 3TU (e-mail: H.Fang@lboro.ac.uk).
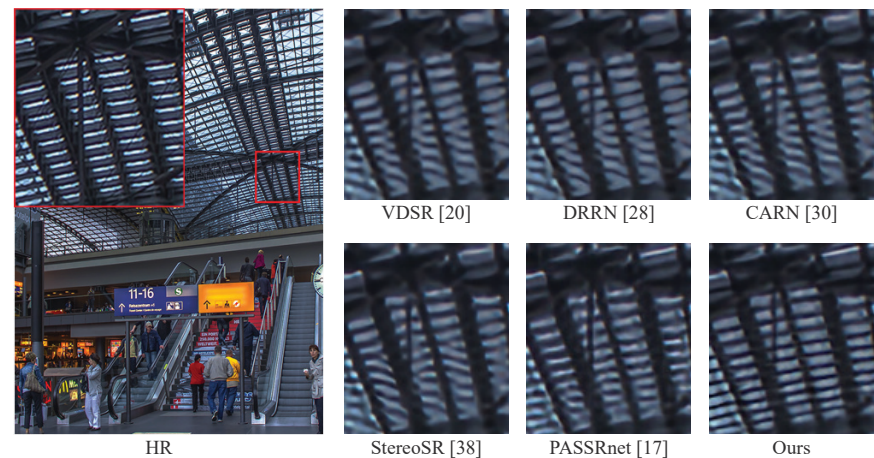

Fig. 1. Image "035" from Flickr1024 test for $\times 4$ SR. The left picture is the original image. We zoom the key part in the image with a red rectangle for clearer comparison. Compared with the state-of-the-art methods, our method synthesizes finer textures and is the closest to HR image.

be categorized into reference-based SR (RefSR), video SR (VSR) and stereo image SR. RefSR [12], [13] matches similar content from additional high resolution images to ensure better recovery of high frequency details. VSR [14], [15] exploits temporal information by aligning frames in a video sequence to reconstruct HR frames. Different from these two types of methods, stereo image SR methods utilize additional features provided in an image pair from a stereoscopic scene to enhance the spatial resolution of the pair. For instance, Jeon et al. [16] learned a parallax prior from stereo image datasets by jointly training two-stage networks. Wang et al. [17] introduced a parallax-attention mechanism with a global receptive field along the epipolar line. Song et al. [18] proposed a self and parallax attention mechanism to reconstruct high-quality stereo image pairs.

Despite the impressive performance achieved by these newly proposed stereo image SR methods (as shown in Fig. 1), rich holistic feature representations from cross view images have remained largely unexplored. As these stereo image SR methods assume that additional features are only derived from stereo correspondences along the epipolar line (as illustrated in Fig. 2), many useful cross view features with similar patterns and textures on both views are ignored in the HR generation process. Thus, it remains an interesting research question how to effectively utilize both global and local features from stereo image pairs to further improve the stereo image SR reconstruction quality.

In this paper, to answer the aforementioned research question, we propose a novel method to incorporate rich feature representations from both holistic views and epipolar lines to enhance the performance of stereo image super-resolution. 

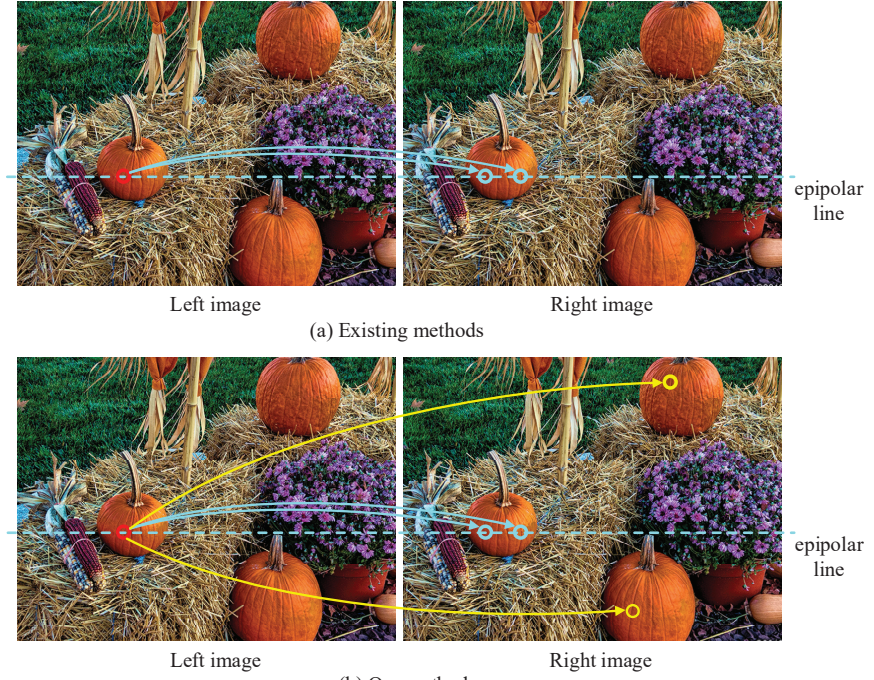

(b) Our method

Fig. 2. Comparison between our method and existing methods. The blue represents the contribution of the right view to the left view along the epipolar line. The yellow stands for the contribution apart from the epipolar line.

Specifically, we propose two innovative mechanisms, including a cross view block (CVB) and spatial perception module (SPM), to integrate cross view spatial features from both global and local perspectives to achieve the goal. With the help of these two types of modules, the newly designed endto-end SR framework, illustrated in Fig. 3, is capable of incorporating more diversified feature representations from different image spatial levels of the two views to enhance the SR reconstruction quality.

The main contributions of this paper are highlighted as follows:

- We propose a novel end-to-end CNN based stereo image SR method that exploits both global and local cross view features to outperform the state-of-the-art (SOTA) stereo image SR methods on several standard benchmark datasets, including the Middlebury, KITTI2012, KITTI2015, and Flickr1024 datasets.

- We propose a cross view block to capture features from different spatial levels across the two different views. To the best of our knowledge, this paper is the first to incorporate both local and global cross view information in stereo image super-resolution.

- A cascaded spatial perception module is designed to further extract efficient hierarchical features by redistributing feature representations at each pixel based on its weights it occupies.

- The last but not the least, our CVCnet performs favorably against the state-of-the-art SR methods in terms of both reconstruction quality and efficiency, especially considering the execution time.

The rest of this paper is organized as follows. In Section II, we briefly review the related works. In Section III, we describe the proposed method in detail. In Section IV, experimental results are performed to demonstrate that our method outperforms the SOTAs. Finally, we conclude this paper in Section V.

\section{RELATED WORK}

In this section, we briefly review the related work. Since CNN-based methods have achieved remarkable performance in recent years, we mainly introduce the CNN-based method.

\section{A. Single Image Super-Resolution}

Since CNN was introduced to SISR in the SRCNN model [19], various deep learning based methods have been proposed to improve the SISR performance. Among these methods, many are designed to extract diverse feature representations from the input low-resolution images. Kim et al. [20] proposed a 20-layer depth model VDSR with the help of residual learning [21] to enlarge receptive fields for capturing contextual information by increasing the depth of the network. Huang et al. [22] used a dense connection architecture to build SRDenseNet so that features extracted from each layer of the CNN could be reused for HR reconstruction. Lai et al. [23] generated high-resolution images with a Laplacian pyramid network so that undesired artifacts could be alleviated by this progressive model. Haris et al. [24] proposed DBPN to utilize iterative up- and down- sampling layers to provide an error feedback mechanism to project errors at each stage. This design ensures an excellent performance when dealing with large-scale image super-resolution tasks. Consequently, DBPN won the NTIRE2018 Super-Resolution Challenge. Guo et al. [25] proposed DepthSR-Net to automatically infer a high-resolution (HR) depth map from its low-resolution (LR) version by hierarchical features driven residual learning. More recently, Mei et al. [26] proposed the cross-scale non-local attention module with integration into a recurrent neural network. Liu et al. [27] introduced a residual feature aggregation framework to extract hierarchical residual features to boost the performance.

In addition to improve HR image quality, many other algorithms seek to enhance the efficiency of their network architectures. Tai et al. [28] proposed DRRN, which strives for deep yet concise networks by exploiting both recursive learning and residual learning. Lim et al. [29] proposed the EDSR by removing unnecessary modules from the conventional ResNet. Ahn et al. [30] invented the CARN model, which implements a cascading mechanism on a residual network to reduce the computational burden. Similarly, Hui et al. [31] proposed IMDN by constructing cascaded information multi-distillation blocks to build an efficient model.

Despite the consistently improved performance, SISR cannot utilize the complementary information of different views in stereo image pairs, which limits the performance in stereo image super-resolution.

\section{B. Reference-Based Image Super-Resolution}

Compared with single image super-resolution, RefSR utilizes rich texture details from a high resolution reference image that shares a similar content of the LR image for HR generation. In one of the most pioneering RefSR methods [32], Zheng et al. designed a cross-scale correspondence network (CC-Net) to match features between LR images and reference 
images and a super-resolution synthesis network (SS-Net) to stack HR reference image features from multiple scales for synthesizing HR image. In [33], Zheng et al. further proposed a cross-scale warping $\mathrm{CNN}$, named CrossNet, to alleviate the effect caused by inter-patch misalignment.

Several recent methods are proposed to enhance RefSR performance when LR images share less similarity with HR reference images. Zhang et al. [13] presented SRNTT to formulate RefSR as a neural texture transfer task. This work leverages a feature swapping mechanism to exploit texture information from similar Ref patches when it is difficult to align the LR image with the HR reference image. Yang et al. [12] presented a texture transformer network TTSR. TTSR integrates a hard-attention module and a soft-attention module to ensure that deep feature correspondences could be discovered to improve HR reconstruction quality.

\section{Video Super-Resolution}

Video super-resolution exploits additional information from the temporal dimension of a video to build finer resolution image frames. Early VideoSR methods design a sequential processing pipeline by providing motion compensation and using aligned consecutive frames as input of a CNN based SR network. Kappeler et al. [34] used a convolutional neural network for video super-resolution tasks for the first time and proposed VSRnet. Caballero et al. [35] proposed VESPCN, which investigates spatial-temporal networks to ensure that both spatial and temporal information could be fused more effectively for the task. Sajjadi et al. [36] introduced the frame recurrent VideoSR method named FRVSR to enhance the temporal consistency for progressively building HR videos.

Since VideoSR methods heavily rely on the accuracy of motion estimation and compensation, some new methods are proposed to tackle this limitation. Instead of explicit motion compensation, Jo et al. [37] designed dynamic upsampling filters (DUF) to embed the alignment process into its endto-end SR network implicitly. Wang et al. [15] presented the SOF-VSR network to produce HR optical flows in a coarse-tofine manner so that accurate motion compensation is calculated for the following SR synthesis network.

\section{Stereo Image Super-Resolution}

In contrast to VideoSR techniques which focus on compensating small displacements across consecutive frames, stereo image SR usually enforces epipolar constraints to find large binocular disparity correspondent patterns across the left and right views of a stereo pair for SR. Jeon et al. [38] learned the parallax prior by training a two-stage network. In their work, the first-stage network enhances the spatial resolution of stereo images in luminance, and the second-stage network reconstructs the HR image by super-resolving both luminance and chrominance components. Wang et al. [17] proposed PASSRnet to tackle the large disparity challenge in stereo image SR. When using their own collected dataset Flickr1024 which contains 1024 high-quality stereo image pairs for stereo image SR, it demonstrates that the parallax-attention mechanism in PASSRnet effectively utilizes corresponding cross view features to generate HR stereo images. To further maintain the stereo-consistency in stereo pairs, Song et al. [18] proposed SPAM by extending the parallax-attention mechanism in PASSRnet. Here, SPAM aggregates the information from its own view and the cross view image simultaneously to produce stereo-consistent image pairs. Yan et al. [39] introduced a feature modulation dense Bblock (FMDB) and created a unified framework to achieve both stereo image super-resolution and stereo image deblurring tasks.

Although the abovementioned methods have achieved good results, they are all based on the assumption that the contribution from the cross view image to the SR reconstruction only comes from corresponding features along the epipolar line. Thus, in our proposed work, we utilize cross view feature representations from both local and global levels to further boost the performance of the state-of-the-art stereo image SR methods.

\section{Proposed Method}

In this section, the proposed method is explained in detail. The three subsections describe our proposed network architecture, the cross view block (CVB) and the cascaded spatial perception module (SPM).

\section{A. Network Architecture}

An overview of our proposed cross view capture network (CVCnet) is illustrated in Fig. 3. CVCnet takes the LR stereo image pair as input and super-resolves the left view image. Note that the framework can also be used to super-resolve both image views. In our work, we follow the settings of [16] and [17] to compare the performance on the reconstructed left view images only. Our CVCnet is composed of three integral components, i.e., initial feature extraction (IFE), cross view block (CVB) and spatial perception module (SPM).

In Fig. 3, $I_{\text {left }}$ and $I_{\text {right }}$ denote the left LR input image and the right LR input image respectively. The first step of the proposed network is to extract multi-channel features by using the initial feature extraction block. Here, the IFE block can be formulated as:

$$
\begin{gathered}
F_{\text {left }}=H_{I F E}\left(I_{\text {left }}\right) \\
F_{\text {right }}=H_{I F E}\left(I_{\text {right }}\right)
\end{gathered}
$$

where $F_{\text {left }}$ and $F_{\text {right }}$ represent the extracted initial features from the input stereo image pair. $H_{I F E}(\cdot)$ stands for the initial feature extraction block, which is composed of one convolution layer and two consecutive residual blocks. It is worth noting that the IFE blocks of the left and right LR images share the same weights. After obtaining $F_{\text {left }}$ and $F_{\text {right }}$, they are utilized as the input of our proposed cross view block, where both global and local feature correlations could be effectively learned to enhance the feature representations from the similar patterns in the pair images. This step is formulated as:

$$
F_{C V B}=H_{C V B}\left(F_{\text {left }}, F_{\text {right }}\right)
$$




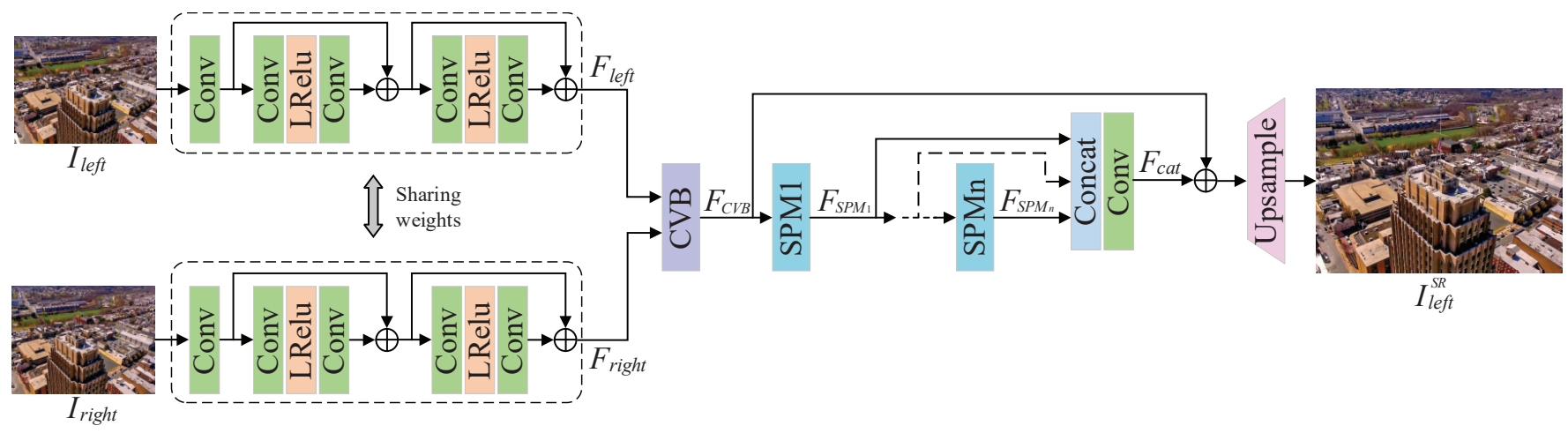

Fig. 3. An overview of CVCnet. $I_{\text {left }}$ and $I_{\text {right }}$ represent the left and right image of an input stereo image pair, respectively. In this paper, we only super-resolve the left image, and $I_{\text {left }}^{S R}$ denotes the reconstructed left image.

where $H_{C V B}(\cdot)$ represents the cross view block and $F_{C V B}$ is the feature captured by the CVB. Then, the $F_{C V B}$ is further processed by the cascaded spatial perception module to effectively extract hierarchical feature representations from the integrated feature of CVB:

$$
\begin{aligned}
F_{S P M_{1}}= & H_{S P M_{1}}\left(F_{C V B}\right) \\
F_{S P M_{2}}= & H_{S P M_{2}}\left(F_{S P M_{1}}\right) \\
& \vdots \\
F_{S P M_{n}}= & H_{S P M_{n}}\left(F_{S P M_{n-1}}\right)
\end{aligned}
$$

where $F_{S P M_{1}}, F_{S P M_{2}}, \ldots$, and $F_{S P M_{n}}$ represent the features extracted by the cascaded spatial perception module. $H_{S P M_{1}}(\cdot), H_{S P M_{2}}(\cdot), \ldots$, and $H_{S P M_{n}}(\cdot)$ represent the related spatial perception module. Then, $F_{S P M_{1}}, F_{S P M_{2}}, \ldots$, and $F_{S P M_{n}}$ are concatenated as:

$$
F_{\text {cat }}=\operatorname{conv}\left(H_{\text {cat }}\left(F_{S P M_{1}}, F_{S P M_{2}}, \ldots, F_{S P M_{n}}\right)\right)
$$

where $H_{\text {cat }}(\cdot)$ represents the concatenation operation and $\operatorname{conv}(\cdot)$ represents the convolution layer with a kernel size of $1 \times 1 . F_{\text {cat }}$ denotes the concatenated feature from the cascaded SPM. Finally, $F_{c a t}$ is utilized to aggregate with the CVB feature to reconstruct the corresponding SR image:

$$
I_{\text {left }}^{S R}=H_{u p}\left(F_{c a t}+F_{C V B}\right)
$$

where $I_{\text {left }}^{S R}$ denotes the reconstructed left image and $H_{\text {up }}(\cdot)$ represents the processing of upsampling. We utilize PixelShuffle [40] to upscale the features to the desired size. $F_{c a t}$ and $F_{C V B}$ are added by residual connection.

\section{B. Cross View Block}

Existing stereo image super-resolution methods rely heavily on features corresponding between left and right views along the epipolar line. However, apart from these local features, global context information provided by cross view images is also useful for the quality enhancement of stereo image SR. Therefore, if we exploit only the local information along the epipolar line, the global information in the stereo image will be wasted. To fully exploit the diverse features from cross

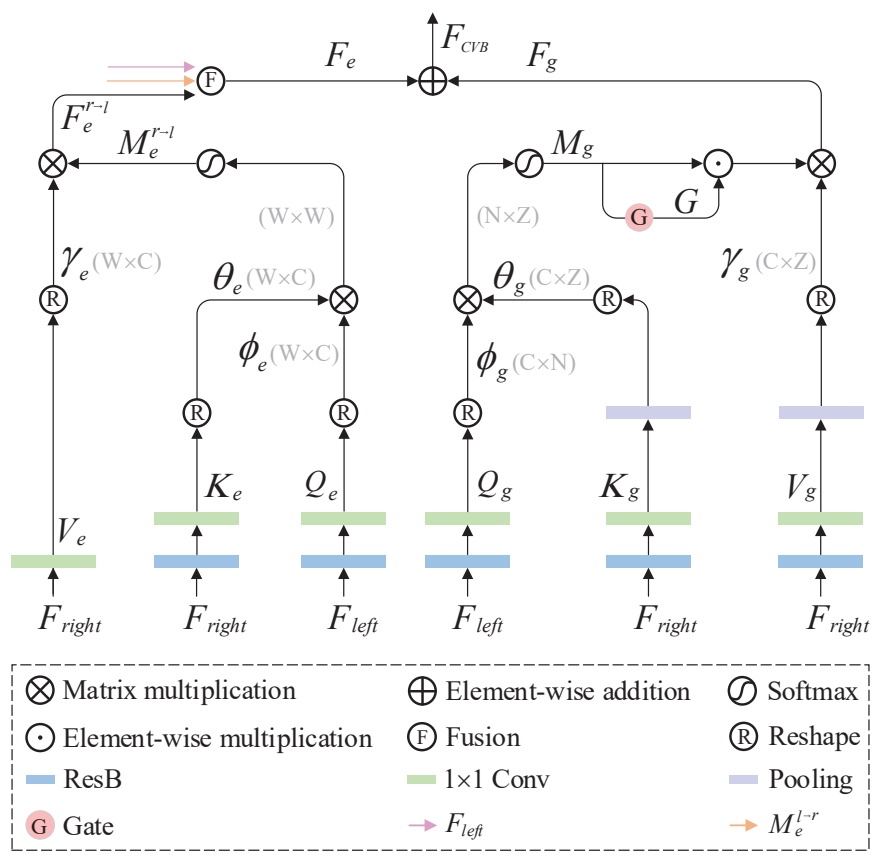

Fig. 4. Overall structure of the cross view block. The similarity matrix is obtained by multiplying $F_{\text {left }}$ and $F_{\text {right }}$, and then the similarity matrix is utilized to refine the right image.

view images, we propose CVB to generate a mechanism that explores both global and local feature correlations to enhance the reconstruction process. As illustrated in Fig. 4, there are two parallel pipelines in the CVB. They form a unified module to explore additional features along the epipolar line and the global context simultaneously.

In the epipolar feature embedding pipeline, a query $Q_{e}$ and a key $K_{e}$ are generated by transforming $F_{\text {left }}$ and $F_{\text {right }}$ via a residual block and convolution layer. Here, the shapes of $Q_{e}$ and $K_{e}$ are $\mathbb{R}^{B \times C \times H \times W}$. To calculate the similarity matrix, $Q_{e}$ and $K_{e}$ are reshaped and flattened to $\phi_{e} \in \mathbb{R}^{B^{\prime} \times W \times C}$ and $\theta_{e} \in \mathbb{R}^{B^{\prime} \times W \times C}$ respectively. It is worth noting that, in Fig. 4, we omit the batchwise dimension, and the same below. By transposing $\theta_{e}$ and multiplying $\phi_{e}$, the similarity matrix of the right view to the left view along the epipolar line is 


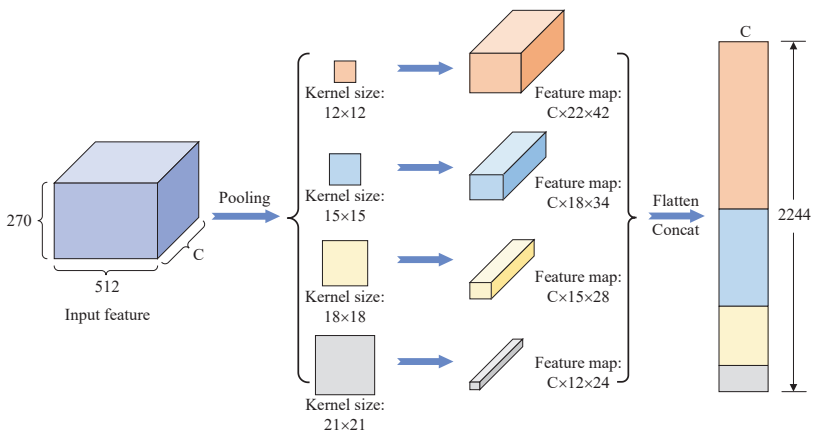

Fig. 5. The process of pooling for $\times 4$ SR during testing. In the testing phase, kernel sizes of the four pooling layers are set to $12,15,18$ and 21 . We assume that the resolution of the HR image is $2 \mathrm{~K}(1080 \times 2048)$, so the size of the input feature is $270 \times 512$.

determined by using the following equation:

$$
M_{e}^{r \rightarrow l}=\operatorname{softmax}\left(\phi_{e} \otimes \theta_{e}^{\mathrm{T}}\right)
$$

where $M_{e}^{r \rightarrow l} \in \mathbb{R}^{W \times W}$ stands for the similarity matrix of the right view to the left view along the epipolar line. Similarly, $M_{e}^{l \rightarrow r}$ can be obtained in the same way. At the same time, a value $V_{e}$ representing the feature $F_{\text {right }}$ is reshaped to $\gamma_{e} \in$ $\mathbb{R}^{W \times C}$ to generate the feature embedding of the right view $F_{e}^{r \rightarrow l}$ by multiplying $M_{e}^{r \rightarrow l}$ :

$$
F_{e}^{r \rightarrow l}=M_{e}^{r \rightarrow l} \otimes \gamma_{e}
$$

Then $F_{e}^{r \rightarrow l}, M_{e}^{l \rightarrow r}$ and $F_{\text {left }}$ are concatenated to obtain the enhanced epipolar line constrained feature embedding $F_{e}$.

Furthermore, to exploit the global contextual features from the cross view image, $Q_{g}($ Query $), K_{g}($ Key $)$ and $V_{g}($ Value $)$ are generated in a similar manner to the epipolar pipeline. It is worth noting that $F_{\text {right }}$ is treated as $V_{g}$ to exploit the information of the right image. In this global feature embedding pipeline, $Q_{g}$ is flattened to $\phi_{g}$ with the shape of $\mathbb{R}^{C \times N}$, and $K_{g}$ is flattened to $\theta_{g}^{\prime}$ with the shape of $\mathbb{R}^{C \times N}$. The global similarity matrix can be formulated as:

$$
M_{n o n-l o c a l}=\operatorname{softmax}\left(\phi_{g}^{\mathrm{T}} \otimes \theta_{g}^{\prime}\right)
$$

where $M_{\text {non-local }} \in \mathbb{R}^{N \times N}$ stands for the similarity matrix of non-local feature representations. As has been described in [41]-[43], the global similarity matrix requires prohibitive computation cost due to the complexity of the matrix multiplication which is $O\left(C N^{2}\right)=O\left(C H^{2} W^{2}\right)$. Thus, in our work, we adopt the pyramid sampling scheme proposed in [42] to reduce the time complexity significantly. Assuming we have $Z$-dimension samples extracted from original response maps for similarity calculation, the time complexity of the matrix multiplication is $O(C N Z)$. Consequently, it is $\frac{Z}{N}$ of the computation in Eq. 9. As illustrated in Fig. 5, if the resolution of the generated HR image is $2 \mathrm{~K}$ and the size of its corresponding input feature map is $270 \times 512$, the time complexity is reduced by $\frac{N}{Z}=\frac{270 \times 512}{2244} \approx 62$ times. Note that $Z$ is controlled by the pooling kernel sizes illustrated

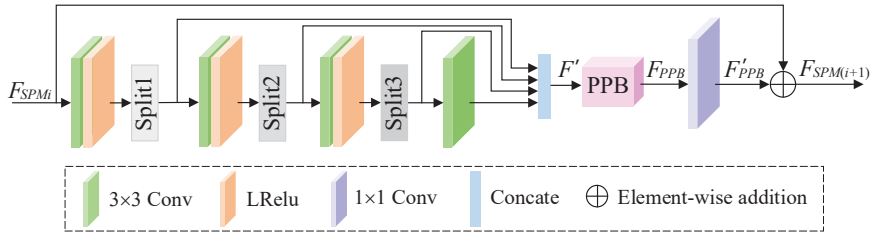

Fig. 6. The architecture of the spatial perception module. The output of the $i$ th SPM is utilized as the input of the $i+1$ th SPM.

in Fig. 5. When the kernel sizes increase, fewer samples are selected to calculate the feature embedding. An ablation study is conducted in our experiments to find the optimal configuration of kernel size to balance the computational complexity and feature performance. The similarity of global feature embedding is expressed as:

$$
M_{g}=\operatorname{softmax}\left(\phi_{g}^{\mathrm{T}} \otimes \theta_{\mathrm{g}}\right)
$$

where $\theta_{\mathrm{g}} \in \mathbb{R}^{C \times Z}$ represents the refined feature from $K_{g}$, $M_{g} \in \mathbb{R}^{N \times Z}$ stands for the global similarity matrix of the right view to the left view.

However, if we directly utilize this similarity matrix $M_{g}$, it may embed too much irrelevant information from the cross view image, which limits the performance. For example, in Fig. 2, in addition to the correspondences across the epipolar line, some parts of the image share similar texture with the target region, but most other parts in the cross view image have no contribution to the SR of this region. Thus, it is necessary to eliminate the features from locations with low similarity to the target location. In our CVB, we design a mask to filter the features with low similarity values from the cross view image:

$$
G(i, j)=\left\{\begin{array}{l}
1, \text { if } M_{g}(i, j)>\tau \\
0, \text { otherwise }
\end{array}\right.
$$

where $G$ represents the mask with the value of 0 or 1 . If the value of the matrix $M_{g}$ at position $(i, j)$ is larger than $\tau$, it is set to 1 , otherwise, it is set to 0 . Mask $G$ is dot multiplied by the global similarity matrix $M_{g}$, then matrix multiply with the transposed $\gamma_{g}$ and obtains $F_{g}$ :

$$
F_{g}=\left(G \cdot M_{g}\right) \otimes \gamma_{g}^{T}
$$

where $\left(G \cdot M_{g}\right)$ represents the filtered matrix which keeps the higher weights of the global similarity matrix. With this design, the proposed global feature embedding $F_{g}$ contains complementary features in the right view to improve the SR of the left view.

Finally, $F_{e}$ and $F_{g}$ are summed to obtain the output of CVB:

$$
F_{C V B}=F_{e}+F_{g}
$$

where $F_{C V B}$ contains both the information along the epipolar line and the global information between the cross views.

\section{Spatial Perception Module}

We propose the spatial perception module (SPM), shown in Fig. 6, to make full use of the integrated features from cross 


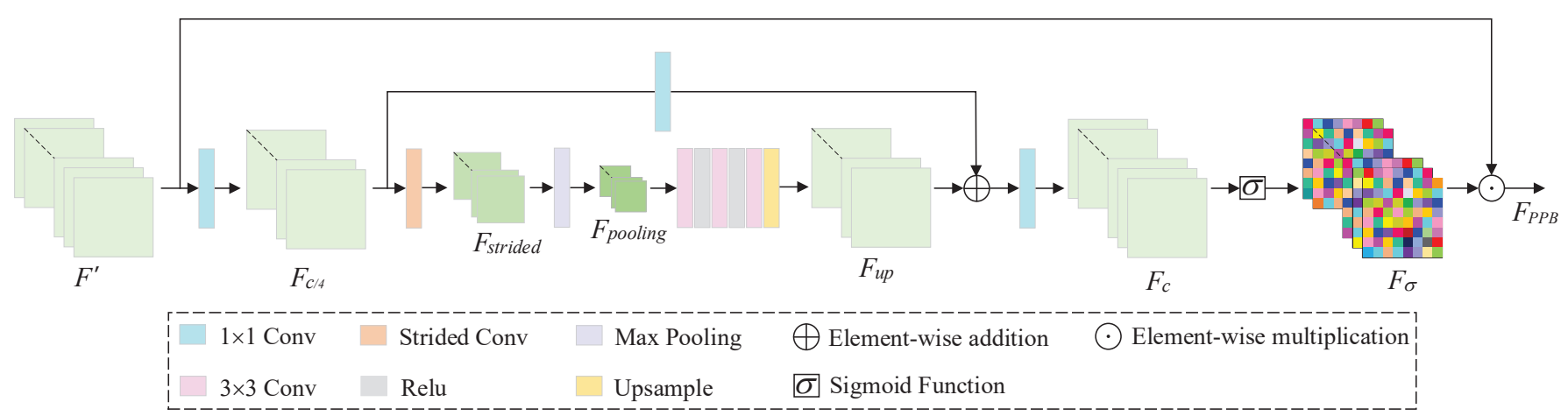

Fig. 7. The architecture of pixel perception block. In PPB, we redistribute each pixel in the feature map according to the weight it occupies.

view images. SPM enhances the useful parts of the features by redistributing each pixel in the feature map according to the weight it occupies. For the input of SPM, a convolution layer with a kernel size of $3 \times 3$ followed by LRelu is utilized to extract the input features. After this step, the channel splitting method used in [31] is applied to the extracted features and these features are divided into two parts according to the channel dimension. One of them is sent to the next feature extraction module, and the other part is preserved for feature fusion. This process can be formulated as:

$$
\begin{aligned}
& F_{1}, F_{1}^{\prime}=\operatorname{Split} 1\left(\operatorname{LRelu}\left(\operatorname{conv}\left(F_{S P M i}\right)\right)\right) \\
& F_{2}, F_{2}^{\prime}=\operatorname{Split} 2\left(\operatorname{LRelu}\left(\operatorname{conv}\left(F_{1}\right)\right)\right) \\
& F_{3}, F_{3}^{\prime}=\operatorname{Split} 3\left(\operatorname{LRelu}\left(\operatorname{conv}\left(F_{2}\right)\right)\right) \\
& F_{4}=\operatorname{conv}\left(F_{3}\right)
\end{aligned}
$$

where $\operatorname{LRelu}(\cdot)$ and $\operatorname{conv}(\cdot)$ represent the activation operation and convolution respectively. Split1(.), Split2(.) and Split3(·) are the channel separation operations that separate the features according to the channel dimension. $F_{1}, F_{2}$ and $F_{3}$ are features that are sent to the next feature extraction module. $F_{1}^{\prime}, F_{2}^{\prime}, F_{3}^{\prime}$ and $F_{4}$ are concatenated as the input of the pixel perception block (PPB):

$$
F^{\prime}=\operatorname{cat}\left(F_{1}^{\prime}, F_{2}^{\prime}, F_{3}^{\prime}, F_{4}\right)
$$

where $\operatorname{cat}(\cdot)$ represents the concatenation operation, $F^{\prime}$ denotes the concatenated feature. Then $F^{\prime}$ is enhanced by the pixel perception block (PPB). The structure of PPB is shown Fig. 7. For the input feature $F^{\prime}$, the number of channels is reduced by a convolution layer with a kernel size of $1 \times 1$ to decrease the consumption of computing resources. Specifically, the number of channels in $F_{c / 4}$ is a quarter of $F^{\prime}$. A progressive method is applied to expand the receptive field of features. First, a strided convolution is utilized and obtains $F_{\text {strided }}$. However, the receptive field of $F_{\text {strided }}$ is still limited, so a max pooling layer is applied and obtains $F_{\text {pooling: }}$ :

$$
F_{\text {pooling }}=\text { pooling }\left(\operatorname{conv}_{s}\left(\operatorname{conv}_{1}\left(F^{\prime}\right)\right)\right)
$$

where $\operatorname{conv}_{1}(\cdot)$ and $\operatorname{conv}_{s}(\cdot)$ represent the convolution with a kernel size of $1 \times 1$ and strided convolution respectively, pooling $(\cdot)$ denotes the max pooling layer, and $F_{\text {pooling }}$ stands for the produced features. Next, a series of convolutions with a kernel size of $3 \times 3$ and activation operations followed by an upsampling layer are applied on $F_{\text {pooling }}$ to obtain $F_{u p}$. From $F_{c / 4}$ to $F_{u p}$, the feature map is compressed and uncompressed in the spatial dimension to extract more structured features based on the global contextual information. Then, $F_{c / 4}$ and $F_{u p}$ are added together, and the number of channels of the feature is restored to $\mathrm{c}$ through the convolution layer with a kernel size of $1 \times 1$. Then, a sigmoid function is used to obtain the weight of each location of the image in the spatial dimension. Finally, $F_{\sigma}$ and $F^{\prime}$ are multiplied in the elementwise dimension to obtain $F_{P P B}$ :

$$
\begin{aligned}
F_{P P B} & =\sigma\left(\operatorname{conv}\left(\operatorname{conv}\left(F_{c / 4}\right)+F_{u p}\right)\right) \odot F^{\prime} \\
& =\sigma\left(F_{c}\right) \odot F^{\prime} \\
& =F_{\sigma} \odot F^{\prime}
\end{aligned}
$$

where $\operatorname{conv}(\cdot)$ stands for the convolution with the kernel size of $1 \times 1, \sigma(\cdot)$ represents the sigmoid function, and $F_{P P B}$ denotes the output of PPB. The obtained feature $F_{P P B}$ is more compact in the spatial dimension, which is informative to the image reconstruction.

After obtaining $F_{P P B}, F_{P P B}$ is sent to a convolution layer with a kernel size of $1 \times 1$ to obtain $F_{P P B}^{\prime}$. Then, $F_{P P B}^{\prime}$ and $F_{S P M i}$ are added by residual connection:

$$
F_{S P M(i+1)}=F_{P P B}^{\prime}+F_{S P M i}
$$

where $F_{S P M(i+1)}$ represents the output of the $i$ th SPM.

\section{EXPERIMENTAL RESULTS}

In this section, experiments are conducted to evaluate our proposed model. Subsection IV-A introduces the datasets and metrics used in our experiments. Then, subsection IV-B presents the implementation details. Next, subsection IV-C compares our model with state-of-the-art methods on different datasets to demonstrate the superiority of the proposed method. Finally, subsection IV-D conducts an ablation study to analyze the contribution from each component to the framework.

\section{A. Datasets and Metrics}

Following [17], we use the high-quality images from the Flickr1024 dataset as our training set. Specifically, there are 
TABLE I

Comparison Results Between Our CVCnet and the State-OF-the-Art Methods. We Compare the PSNR (dB) AND SSIM on the KITTI2015, KITTI2012, MiddLEBURY, AND FLICKR1024 DATASETS.

\begin{tabular}{|c|c|c|c|c|c|c|c|c|c|c|}
\hline \multirow{2}{*}{ Method } & \multirow{2}{*}{ Scale } & \multirow{2}{*}{ Params } & \multicolumn{2}{|c|}{ KITTI2015 } & \multicolumn{2}{|c|}{ KITTI2012 } & \multicolumn{2}{|c|}{ Middlebury } & \multicolumn{2}{|c|}{ Flickr1024 } \\
\hline & & & PSNR & SSIM & PSNR & SSIM & PSNR & SSIM & PSNR & SSIM \\
\hline Bicubic & $\times 2$ & - & 28.0108 & 0.8863 & 28.6406 & 0.8851 & 30.6709 & 0.9013 & 24.9866 & 0.8240 \\
\hline SRCNN [19] & $\times 2$ & $0.07 \mathrm{M}$ & 29.1776 & 0.9104 & 30.0134 & 0.9089 & 32.7338 & 0.9282 & 26.9328 & 0.8800 \\
\hline VDSR [20] & $\times 2$ & $0.64 \mathrm{M}$ & 29.2397 & 0.9115 & 30.1505 & 0.9101 & 33.2151 & 0.9326 & 27.1425 & 0.8849 \\
\hline DRRN [28] & $\times 2$ & $0.29 \mathrm{M}$ & 29.1996 & 0.9100 & 30.0778 & 0.9088 & 33.5858 & 0.9357 & 27.4428 & 0.8907 \\
\hline CARN [30] & $\times 2$ & $1.55 \mathrm{M}$ & 29.3657 & 0.9146 & 30.3224 & 0.9133 & 33.6302 & 0.9375 & 27.7621 & 0.8968 \\
\hline SRFBN [44] & $\times 2$ & $2.04 \mathrm{M}$ & 29.6012 & 0.9182 & 30.5328 & 0.9159 & 34.2830 & 0.9421 & 27.7800 & 0.8976 \\
\hline StereoSR [38] & $\times 2$ & $1.06 \mathrm{M}$ & 29.5407 & 0.9170 & 30.5096 & 0.9154 & 33.9657 & $\overline{0.9400}$ & 28.0497 & 0.9009 \\
\hline PASSRnet [17] & $\times 2$ & $1.30 \mathrm{M}$ & 29.7916 & $\underline{0.9191}$ & 30.7033 & 0.9168 & 34.0420 & 0.9413 & 28.3011 & 0.9044 \\
\hline CPASSRnet & $\times 2$ & $42.39 \mathrm{M}$ & $\overline{29.6650}$ & $\overline{0.9173}$ & $\overline{29.5071}$ & $\overline{0.9053}$ & 33.4020 & 0.9384 & $\overline{27.8661}$ & $\overline{0.8987}$ \\
\hline CVCnet (ours) & $\times 2$ & $0.97 \mathrm{M}$ & 29.9326 & 0.9224 & 30.8684 & 0.9198 & 34.4025 & 0.9450 & 28.4403 & 0.9081 \\
\hline Bicubic & $\overline{\times 3}$ & - & 25.4217 & 0.7903 & 26.1085 & 0.8021 & 27.9159 & 0.8222 & 22.8038 & 0.7081 \\
\hline SRCNN [19] & $\times 3$ & $0.07 \mathrm{M}$ & 26.3797 & 0.8254 & 27.2015 & 0.8342 & 29.4326 & 0.8579 & 23.8811 & 0.7650 \\
\hline VDSR [20] & $\times 3$ & $0.64 \mathrm{M}$ & 26.4891 & 0.8274 & 27.3085 & 0.8355 & 29.7154 & 0.8622 & 23.9374 & 0.7670 \\
\hline DRRN [28] & $\times 3$ & $0.29 \mathrm{M}$ & 26.4781 & 0.8274 & 27.3188 & 0.8354 & 30.0162 & 0.8680 & 24.2901 & 0.7835 \\
\hline CARN [30] & $\times 3$ & $1.55 \mathrm{M}$ & 26.6260 & 0.8336 & 27.4743 & 0.8410 & 30.0432 & 0.8701 & 24.4251 & 0.7882 \\
\hline SRFBN [44] & $\times 3$ & $2.60 \mathrm{M}$ & 26.9019 & 0.8412 & 27.7428 & 0.8471 & 30.7587 & 0.8828 & 24.6438 & 0.7959 \\
\hline StereoSR [38] & $\times 3$ & $1.06 \mathrm{M}$ & 26.6567 & 0.8360 & 27.4890 & 0.8430 & 30.3364 & 0.8762 & 24.5625 & 0.7938 \\
\hline PASSRnet [17] & $\times 3$ & $1.32 \mathrm{M}$ & 26.9567 & 0.8417 & 27.8230 & 0.8487 & 30.4150 & 0.8781 & 24.7509 & 0.8002 \\
\hline CPASSRnet [45] & $\times 3$ & $42.39 \mathrm{M}$ & 26.7910 & 0.8389 & 26.7666 & $\overline{0.8310}$ & 29.9776 & 0.8756 & 24.5181 & 0.7924 \\
\hline CVCnet (ours) & $\times 3$ & $0.98 \mathrm{M}$ & 27.0761 & 0.8457 & 27.8788 & 0.8509 & 30.5618 & 0.8826 & 24.8389 & 0.8039 \\
\hline Bicubic & $\overline{\times 4}$ & 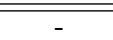 & 23.9042 & $\overline{0.7100}$ & 24.6368 & $\overline{0.7334}$ & 26.3935 & $\overline{\overline{0.7564}}$ & 21.7688 & $\overline{0.6295}$ \\
\hline SRCNN [19] & $\times 4$ & $0.07 \mathrm{M}$ & 24.7179 & 0.7484 & 25.5436 & 0.7683 & 27.6917 & 0.7927 & 22.4584 & 0.6769 \\
\hline VDSR [20] & $\times 4$ & $0.64 \mathrm{M}$ & 24.8784 & 0.7543 & 25.7152 & 0.7731 & 27.9912 & 0.8034 & 22.5828 & 0.6847 \\
\hline SRDenseNet [22] & $\times 4$ & & 24.8753 & 0.7664 & 25.7867 & 0.7840 & 27.9679 & 0.8107 & 22.8992 & 0.7100 \\
\hline DRRN [28] & $\times 4$ & $0.29 \mathrm{M}$ & 24.9179 & 0.7552 & 25.7298 & 0.7730 & 28.0710 & 0.8032 & 22.6757 & 0.6887 \\
\hline CARN [30] & $\times 4$ & $1.55 \mathrm{M}$ & 25.1038 & 0.7644 & 25.9123 & 0.7806 & 28.1301 & 0.8064 & 22.8028 & 0.6962 \\
\hline SRFBN [44] & $\times 4$ & $3.46 \mathrm{M}$ & 25.2839 & 0.7743 & 26.1168 & 0.7889 & 28.7643 & $\underline{0.8248}$ & 23.1519 & 0.7171 \\
\hline DRN-S [46] & $\times 4$ & $4.58 \mathrm{M}$ & 25.3592 & 0.7787 & 26.2796 & 0.7904 & 28.7975 & $\overline{0.8283}$ & 23.3468 & 0.7281 \\
\hline StereoSR [38] & $\times 4$ & $1.06 \mathrm{M}$ & 25.1164 & 0.7679 & 25.9354 & 0.7839 & 28.2417 & 0.8133 & 22.9627 & 0.7075 \\
\hline PASSRnet [17] & $\times 4$ & $1.35 \mathrm{M}$ & 25.3421 & 0.7722 & 26.1772 & 0.7874 & 28.3641 & 0.8153 & 23.0869 & 0.7103 \\
\hline CPASSRnet [45] & $\times 4$ & $42.39 \mathrm{M}$ & 25.1206 & 0.7693 & 25.3146 & 0.7712 & 28.3068 & 0.8194 & 23.0928 & 0.7154 \\
\hline CVCnet (ours) & $\times 4$ & $0.99 \mathrm{M}$ & 25.5489 & 0.7801 & 26.3481 & 0.7935 & 28.6479 & 0.8231 & 23.2231 & 0.7192 \\
\hline
\end{tabular}

800 pairs of images used for training, 112 pairs for validation and 112 pairs for testing in Flickr1024. For a fair comparison with the benchmark models, all the models, including our proposed model, are trained by using 800 pairs of images from the Flickr1024 dataset. During the testing phase, we present the results based on the following test sets: 5 image pairs from the Middlebury [47] dataset, 20 image pairs from the KITTI2012 [48] dataset, 20 image pairs from the KITTI2015 [49] dataset and 112 image pairs from Flickr1024. For the quantitative evaluation, both the peak signal-to-noise ratio (PSNR) [50] and structural similarity index (SSIM) [51] are used as the evaluation metrics.

\section{B. Implementation Details}

During training, we synthesize the LR images by downsampling the training HR images by bicubic interpolation. Following [17], these LR images are cropped to $30 \times 90$ patches with a stride of 20 . At the same time, corresponding HR images are also cropped to match the change in image size. For data augmentation, these patches are randomly flipped horizontally and vertically. In our work, we use ADAM with $\beta 1=0.9, \beta 2=0.999$ as the optimizer of the model. We train our model for 80 epochs with a batch size of 16 , and the initial learning rate is set to 0.0002 . The learning rate is reduced by half every 30 epochs. The L2 (mean square error) loss is used as the total loss. We empirically set $\tau$ defined in Section III to 0.009. All experiments are performed under the environments of NVIDIA GeForce RTX 2080Ti GPUs and an Intel i9-10940X processor @3.30 GHz.

\section{Comparisons With State-of-the-Art Methods}

In this section, we compare our method with several stateof-the-art CNN-based single image SR methods (i.e., SRCNN [19], VDSR [20], SRDenseNet [22], DRRN [28], CARN [30], SRFBN [44], DRN-S [46]) and three stereo image SR methods (i.e., StereoSR [38], PASSRnet [17], CPASSRnet [45]). For fair comparison, we use the codes provided by the authors in the above methods and retrain them by the same training set. For the SISR methods, the inputs are single images, so we split the paired stereo images into two individual images for the training of SISR methods. Here, we use the low resolution images as the input and the corresponding high resolution images as the ground truth to supervise the training of the SISR methods. Following [16], [17], EDSR [29], RCAN [52] and SAN [53] are not included in our comparison since their model sizes are much larger than ours. During testing, all tests 


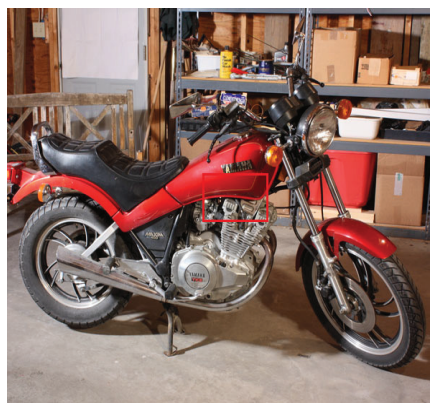

Motorcycle from Middlebury

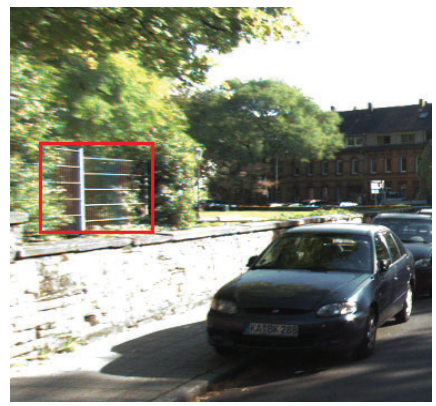

011 from KITTI2012

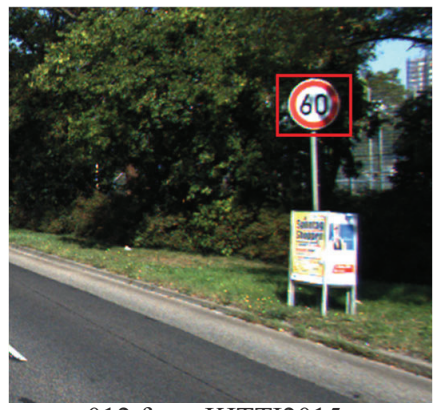

012 from KITTI2015

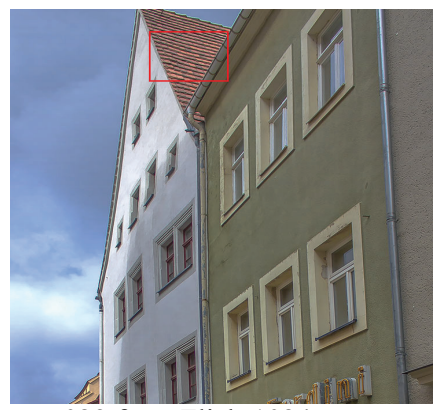

029 from Flickr1024 test

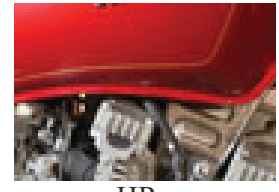

HR

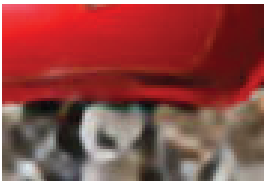

CARN
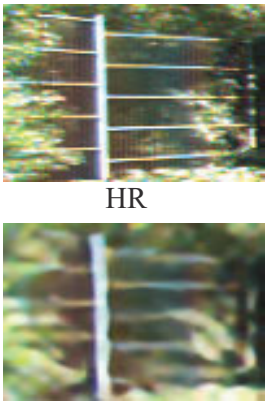

CARN

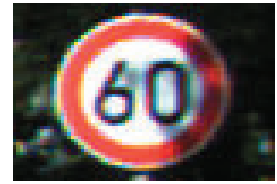

HR

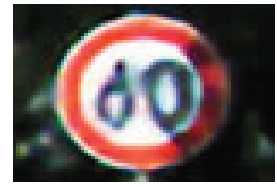

CARN

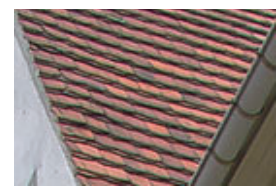

HR

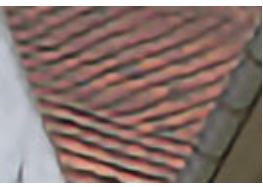

CARN

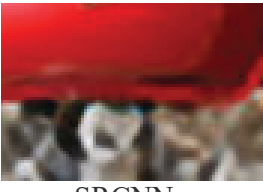

SRCNN

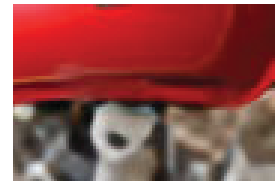

StereoSR

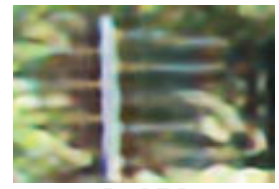

SRCNN

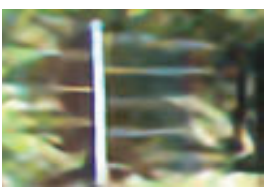

StereoSR

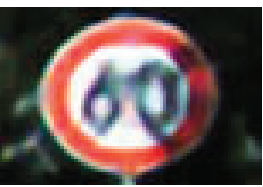

SRCNN

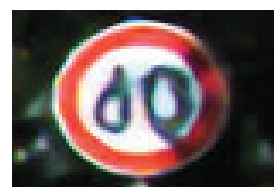

StereoSR

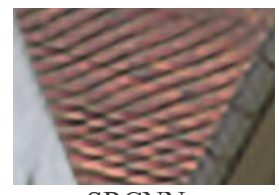

SRCNN

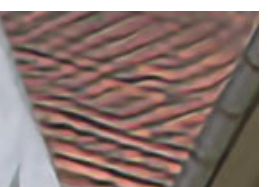

StereoSR

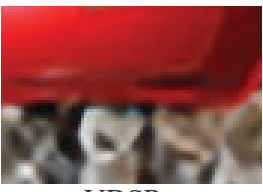

VDSR

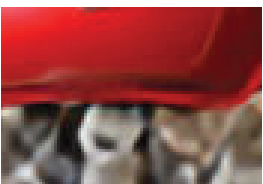

PASSRnet

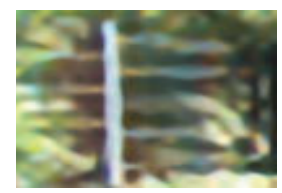

VDSR

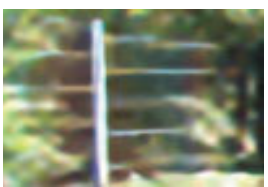

PASSRnet

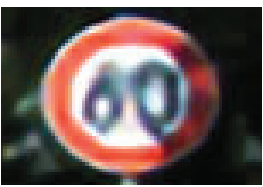

VDSR

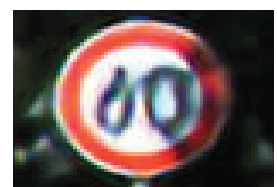

PASSRnet

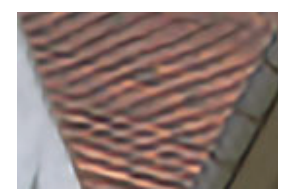

VDSR

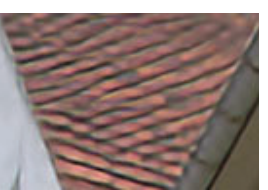

PASSRnet

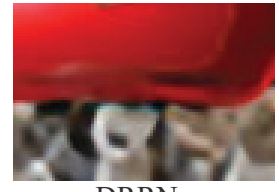

DRRN

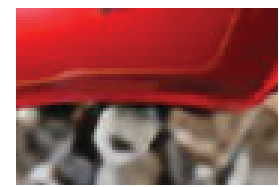

Ours

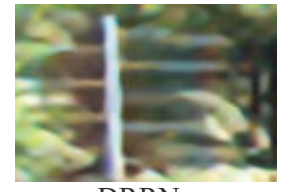

DRRN

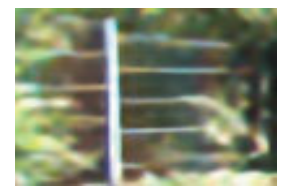

Ours

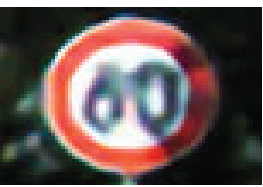

DRRN

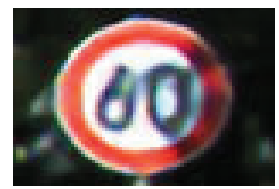

Ours

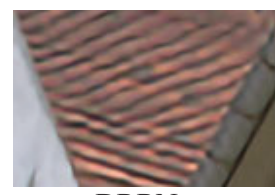

DRRN

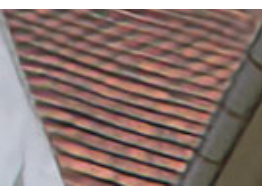

Ours

Fig. 8. Visual comparisons of the state-of-the-art methods and our model on different benchmark test datasets for $\times 4$ SR. We crop the original left image in stereo image pairs to a uniform size and show it on the left. The key contrast parts in the red rectangle are magnified to display on the right.

are performed on the Middlebury, KITTI2012, KITTI2015 and Flickr1024 benchmark test sets.

1) Quantitative Evaluation: The quantitative evaluation of different models on the standard test sets is shown in Table I. In addition to the commonly used test sets Middlebury, KITTI2012 and KITTI2015, we further add the test set in Flickr1024 for comparison. It can be seen from Table I that the models with better performance in single image superresolution still achieve better performance in stereo image super-resolution. For example, CARN has better performance on SISR compared to DRRN, and its PSNR and SSIM values are still higher than those of DRRN on stereo images. In contrast to the SISR methods, the stereo image superresolution methods effectively use the information from cross view images; thus, they help the reconstruction of the target image. Specifically, we can see that StereoSR utilizes the information of the right image, which makes it have a better performance than CARN while the parameter is two-thirds of CARN. From the Table I it is found that, although the parameters of SRFBN and DRN-S are about 4 times that of 


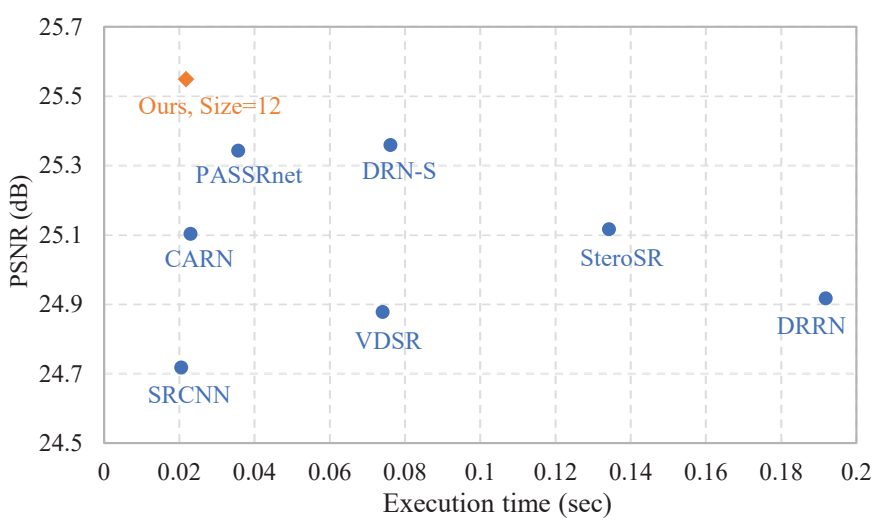

Fig. 9. Trade-off between performance and execution time on the KITTI2015 dataset for $\times 4$ SR. SRFBN and CPASSRnet are not included in the figure due to their long execution time $(0.2002$ s and 0.4435 s respectively). "Size" denotes the smallest size of pooling kernel in CVB. For example, Size $=12$ means that the sizes of the four pooling kernels are 12, 15, 18 and 21.

our model, in overall, our method has a better performance compared to SRFBN and a comparable performance to DRN-

S. Compared with these SOTA methods, our model explores more feature representations across the left and right images. It is also worth noting that compared with PASSRnet, we still have better performance while the number of parameters is reduced by a quarter.

2) Qualitative Evaluation: Furthermore, we compare the visual effects of our model with the state-of-the-art methods on four test sets. The comparison results are illustrated in Fig. 8. We crop the image to compare the key part in each image in detail by framing it with a red rectangle. As shown in Fig. 8, there is a large visual gap between the image produced by most methods and the HR image; however, the image processed by our method has the best reconstruction quality.

As shown in "Motorcycle", the two edges of the motorcycle in the HR image are clear and sharp. However, the contour reconstructed by most methods has difficulty identifying sharp edges. For example, VDSR and DRRN almost lose the information of the bottom contour. In the image reconstructed by PASSRnet, the edges and detailed texture are still very blurry. In contrast to the SOTA methods, our method produces images with sharper outlines that are most similar to the HR image.

We can see from "011" that most SISR methods produce very blurry images. Specifically, the image produced by SRCNN has difficulty identifying railings in the middle. Although existing stereo image super-resolution methods surpass the SISR methods, they still cannot reconstruct the middle railings in the image. Our method not only reconstructs the railing in the middle of the image more clearly, but also has richer details and good visual effects.

As displayed in "012", for the number " 60 " in the image, most methods have failed to produce a clear visual effect. For example, the number generated by DRRN is incomplete. Although StereoSR and PASSRnet can produce clearer textures, for the digit " 6 ", they produce a deformed visual effect, which makes it difficult to even recognize the digit. The image produced by our method not only has the richest details but also reconstructs the meaningful information in the original image.

For "029", we can see that the lines of the tiles in the HR image are neat and clear. The image produced by SRCNN not only has a very blurry effect but also the direction of all the lines is inconsistent with the HR image. CARN has a better visual effect than SRCNN; however, the details are still not clear enough. Although PASSRnet produces sharper textures, most of its lines are still far from those of the HR image. Compared with other methods, our method produces a clearer outline. More importantly, the tile lines are independent of each other and have the same direction, which is the most similar to the HR image.

3) Execution Time: In this section, we study the tradeoff between the execution time and performance of different methods. As we can see from Fig. 9, although StereoSR has a slightly better performance than CARN, it requires a long execution time. Despite the results achieved by DRN-S is the closest to our method, its execution time is approximately 4 times longer than that of our CVCnet. Compared with StereoSR, PASSRnet not only has a better performance but also executes faster. It is encouraging that the size of the pooling kernel has little effect on the performance of our model. When the size of the pooling kernel is set to 12, our model surpasses PASSRnet by a large margin in both performance and running speed. It also means that we can flexibly achieve the balance between the performance and execution speed by adjusting the size of the pooling kernel.

\section{Model Analysis}

Ablation experiments on different datasets are conducted to analyze the components in the CVCnet framework. In this study, we retrain a set of variant models by using the same implementation configuration of the CVCnet as described in subsection IV-B.

1) Cross View Block: As discussed in the previous sections, the CVB is designed to explore additional features from global contextual patterns and along the epipolar line in the cross view image. As shown in Table II, we remove the CVB in model 4 to evaluate its contribution to the overall performance. Compared with model 6, which has the CVB in the framework, it is found that CVB significantly improves the performance of the proposed model. In particular, on the test set of KITTI2015, the PSNR value is improved from $25.3702 \mathrm{~dB}$ to $25.5489 \mathrm{~dB}$ when embedding the CVB into the architecture. To further prove that the improved performance is not caused by simply adding more parameters, we present a model variant (model 2 in Table III) by adding a convolution layer that has the same number of increased parameters as when including the CVB. As shown in Table III, the performance of model 2 does not benefit from simply adding more convolution layers into the architecture. Thus, these two model variants demonstrate the effectiveness of our proposed CVB.

2) Mask in CVB: The mask in CVB is proposed to avoid feature biases incurred by using irrelevant feature representations from cross view images. To demonstrate the role of the proposed mask, we present a model variant (model 3 in Table III) by removing the mask from the proposed 
TABLE II

Comparison Results Achieved by OUR Method and Its Variants. All the Variants are Retrained AND Test the PSNR (dB) ANd SSIM ON KITTI2015 DATASETS FOR $\times 4$ SR.

\begin{tabular}{|c||cccccc|}
\hline & 1 & 2 & 3 & 4 & 5 & 6 \\
\hline IFE & $\checkmark$ & & $\checkmark$ & $\checkmark$ & $\checkmark$ & $\checkmark$ \\
Sharing weights & $\checkmark$ & $\checkmark$ & $\checkmark$ & $\checkmark$ & $\checkmark$ & $\checkmark$ \\
CVB & & $\checkmark$ & $\checkmark$ & $\checkmark$ & & $\checkmark$ \\
SPM & & & $\checkmark$ & & & $\checkmark$ \\
\hline KITTI2015 & $25.2937 / 0.7694$ & $25.4413 / 0.7768$ & $25.4683 / 0.7783$ & $25.3702 / 0.7747$ & $25.3941 / 0.7752$ & $\mathbf{2 5 . 5 4 8 9 / 0 . 7 8 0 1}$ \\
\hline
\end{tabular}

TABLE III

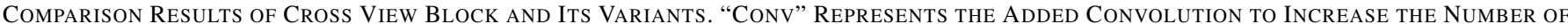
Parameters, the Same Below. The PSNR (dB) and SSIM Value Are Tested on KITTI2015, KitTi2012 and Middlebury Datasets RESPECTIVELY FOR $\times 4$ SR.

\begin{tabular}{|l|l|c|c|c|c|}
\hline \multirow{2}{*}{ Models } & \multirow{2}{*}{ Params } & KITTI2015 & KITTI2012 & Middlebury \\
\cline { 3 - 5 } & & PSNR/SSIM & PSNR/SSIM & PSNR/SSIM \\
\hline \hline 1 & CVCnet w/o CVB & $988 \mathrm{~K}$ & $25.3702 / 0.7747$ & $26.2147 / 0.7898$ & $28.3990 / 0.8182$ \\
2 & CVCnet w/o CVB + Conv & $1036 \mathrm{~K}$ & $25.3567 / 0.7744$ & $26.2163 / 0.7886$ & $28.4284 / 0.8179$ \\
3 & CVCnet w/o Mask & $1016 \mathrm{~K}$ & $25.4622 / 0.7769$ & $26.2654 / 0.7908$ & $28.5444 / 0.8189$ \\
4 & CVCnet & $1016 \mathrm{~K}$ & $\mathbf{2 5 . 5 4 8 9 / 0 . 7 8 0 1}$ & $\mathbf{2 6 . 3 4 8 1 / 0 . 7 9 3 5}$ & $\mathbf{2 8 . 6 4 7 9 / 0 . 8 2 3 1}$ \\
\hline
\end{tabular}

TABLE IV

The Effect of Different Sizes of Pooling Kernel on AVerage Inference Time (SECond), Memory Consumption (MB) And Performance. In This Table, "Size” Represents the Size of Pooling Kernel. For Example, Size $=(3,6,9,12)$ Means That the Sizes of the Four Pooling Kernels Are 3, 6, 9 And 12. The PSNR (dB) AND SSIM Value ARe Tested on the KITTI2015 Dataset For $\times 4$ SR.

\begin{tabular}{|c|c|c|c|c|c|}
\hline & Ours w/o CVB & Size $=(3,6,9,12)$ & Size $=(6,9,12,15)$ & Size $=(9,12,15,18)$ & Size $=(12,15,18,21)$ \\
\hline \hline Time & 0.01596 & 0.03281 & 0.02566 & 0.02368 & 0.02180 \\
Memory & 1088 & 2872 & 1540 & 1244 & 1164 \\
PSNR/SSIM & $25.3702 / 0.7747$ & $\mathbf{2 5 . 5 4 8 9 / 0 . 7 8 0 1}$ & $\mathbf{2 5 . 5 4 8 9 / 0 . 7 8 0 1}$ & $\mathbf{2 5 . 5 4 8 9 / 0 . 7 8 0 1}$ & $25.5488 / 0.7801$ \\
\hline
\end{tabular}

TABLE V

Comparison Results of Spatial Perception Module and Its Variants. The PSNR (dB) and SSim Value Are Tested on KitTi2015, KITTI2012 AND MIDDLEBURY DATASETS RESPECTIVELY FOR $\times 4$ SR.

\begin{tabular}{|c|l|c|c|c|c|}
\hline \multirow{2}{*}{ Models } & \multirow{2}{*}{ Params } & KITTI2015 & KITTI2012 & Middlebury \\
\cline { 3 - 5 } & & PSNR/SSIM & PSNR/SSIM & PSNR/SSIM \\
\hline \hline 1 & CVCnet w/o SPM & $557 \mathrm{~K}$ & $25.3941 / 0.7752$ & $26.1972 / 0.7893$ & $28.4878 / 0.8185$ \\
2 & CVCnet w/o SPM + Conv & $1205 \mathrm{~K}$ & $25.3022 / 0.7696$ & $26.0707 / 0.7841$ & $28.3001 / 0.8104$ \\
3 & CVCnet w/o PPB & $948 \mathrm{~K}$ & $25.3583 / 0.7749$ & $26.1772 / 0.7898$ & $28.4264 / 0.8200$ \\
4 & CVCnet w/o PPB + Conv & $1164 \mathrm{~K}$ & $25.3689 / 0.7765$ & $26.2164 / 0.7914$ & $28.5224 / 0.8209$ \\
5 & CVCnet & $1016 \mathrm{~K}$ & $\mathbf{2 5 . 5 4 8 9 / 0 . 7 8 0 1}$ & $\mathbf{2 6 . 3 4 8 1 / 0 . 7 9 3 5}$ & $\mathbf{2 8 . 6 4 7 9 / 0 . 8 2 3 1}$ \\
\hline
\end{tabular}

architecture. As shown in Table III and Fig. 10, the average PSNR value on the KITTI2015 dataset is improved by 0.1 $\mathrm{dB}$ with the designed mask. This indicates that the mask is capable of filtering out irrelevant feature representations for better SR reconstruction.

3) Different Sizes of Pooling Kernel in CVB: During training, we compress $K_{g}$ and $V_{g}$ in Fig. 4 by pooling operations to reduce computational cost. Here, the kernel sizes of the four pooling layers are 3, 6, 9, and 12 for the pyramid sampling process. When larger kernel sizes are applied to the global contextual feature embedding pipeline, the computational cost can be reduced further. In the testing phase, we investigate the impact of different kernel sizes and their performances. As shown in Table IV, we compare four model variants with different sets of kernel sizes. It can be seen from Table IV that the smaller the pooling kernels, the more time and memory it consumes. Specifically, when kernel sizes are set to (3, $6,9,12)$, the time and memory consumed by CVCnet are approximately three times as much as those of the model without CVB. In contrast, when kernel sizes are set to (12, $15,18,21)$, the time and memory consumed by CVCnet are decreased significantly. It is worth noting that the performance is stable even when the size of the pooling kernels increases. This demonstrates that our model is insensitive to the size of the pooling kernels during testing.

4) Spatial Perception Module: We propose the cascaded spatial perception module to make use of the integrated features from cross view images more effectively. In this study, a model variant (model 5 in Table II) is built to test the proposed framework without SPM. Based on the experimental results on the KITTI2015 dataset, it is found that the SPM is useful to improve the overall performance of the proposed network architecture. As highlighted in the CVB ablation study, the parameter number is reduced when we remove a module from the proposed framework. Thus, we conduct experiments to demonstrate that the improved performance is not caused by 
TABLE VI

The Impact of Different InPuts on the Performance. The PSNR (dB) And SSIM Value ARe Tested on KITTI2015, KITTI2012 And MidDLEBURY DATASETS RESPECTIVELY FOR $\times 4$ SR.

\begin{tabular}{|l|c|c|c|c|}
\hline \multirow{2}{*}{ Models } & \multirow{2}{*}{ Inputs } & KITTI2015 & KITTI2012 & Middlebury \\
\cline { 3 - 5 } & & PSNR/SSIM & PSNR/SSIM & PSNR/SSIM \\
\hline \hline CVCnet with single input & Left & $25.3570 / 0.7748$ & $26.1940 / 0.7892$ & $28.4554 / 0.8187$ \\
CVCnet with replicated inputs & Left + Left & $25.4155 / 0.7767$ & $26.2185 / 0.7904$ & $28.4605 / 0.8187$ \\
CVCnet & Left + Right & $\mathbf{2 5 . 5 4 8 9 / 0 . 7 8 0 1}$ & $\mathbf{2 6 . 3 4 8 1 / 0 . 7 9 3 5}$ & $\mathbf{2 8 . 6 4 7 9 / 0 . 8 2 3 1}$ \\
\hline
\end{tabular}

TABLE VII

THE IMPACT OF Different LoSSES ON THE PERFormance. THE PSNR (dB) AND SSIM VAlue ARE TeSTED ON KITTI2015, KITTI2012 AND MIDDLEBURY DATASETS RESPECTIVELY FOR $\times 4$ SR.

\begin{tabular}{|c|c|c|c|}
\hline \multirow{2}{*}{ Losses } & KITTI2015 & KITTI2012 & Middlebury \\
\cline { 2 - 4 } & PSNR/SSIM & PSNR/SSIM & PSNR/SSIM \\
\hline \hline SSIM & $25.4497 / 0.7784$ & $26.2421 / 0.7923$ & $28.4071 / 0.8216$ \\
MSSIM & $25.4181 / 0.7877$ & $26.2394 / 0.8010$ & $28.6080 / 0.8311$ \\
L1 & $25.2944 / 0.7751$ & $26.1866 / 0.7910$ & $28.6068 / 0.8223$ \\
L2 & $\mathbf{2 5 . 5 4 8 9 / 0 . 7 8 0 1}$ & $\mathbf{2 6 . 3 4 8 1 / 0 . 7 9 3 5}$ & $\mathbf{2 8 . 6 4 7 9 / 0 . 8 2 3 1}$ \\
\hline
\end{tabular}

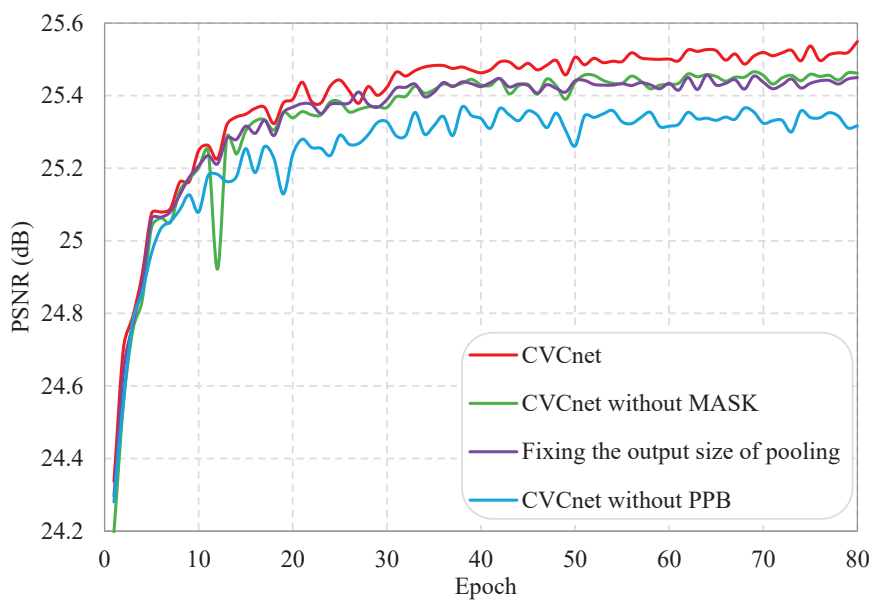

Fig. 10. The PSNR curves of training between different models. We retrain all the models and test them on KITTI2015 dataset for $\times 4$ SR.

simply adding more parameters from the SPM. Similar to the experiments for CVB, we add a convolution layer to ensure that the parameter number of the model variant without SPM is more than the number of the proposed model. In addition, we evaluate the networks with and without PPB. As shown in Table V, the results demonstrate that the SPM, especially the PPB module in the architecture, is important for performance improvement.

5) Different Inputs: Stereo image super-resolution utilizes additional information from cross view images to enhance the performance compared with SISR. To test the effectiveness of our model, we present the results from two more model variants in Table VI. One is with the input of a single view on the left image, and the other is with replicated left images. All the results are presented based on testing on the KITTI2015, KITTI2012 and Middlebury datasets. As shown in Table VI, the PSNR values of model variant 1 with the input of the left view only are $25.3570 \mathrm{~dB}, 26.1940 \mathrm{~dB}$ and $28.4554 \mathrm{~dB}$, and the PSNR values of model variant 2 with the input of the replicated left view are $25.4155 \mathrm{~dB}, 26.2185 \mathrm{~dB}$ and $28.4605 \mathrm{~dB}$, respectively. In contrast, CVCnet with cross view input improves the performance to $25.5489 \mathrm{~dB}, 26.3481$ $\mathrm{dB}$ and $28.6479 \mathrm{~dB}$. The improvement from the cross view input is statistically significant, which is much better than the results from model variant 2 with replicated view input. These experiments show that our CVCnet can effectively capture the information from different views.

6) Sharing Weights: Due to the strong similarity between cross view images, the initial feature extraction module of our model adopts the method of sharing weights for training. As shown in Table II, compared with model 3, which does not share weights, model 6 with shared weights has a better PSNR value on KITTI2015, which is $25.5489 \mathrm{~dB}$. This indicates that the weight sharing strategy alleviates the overfitting issue during the training and facilitates the fusion of feature information between the left and right images.

7) Initial Feature Extraction Module: We also verify the effectiveness of the initial feature extraction module (IFE) in our CVCnet. As we can see from Table II, compared with model 2 which uses simple convolution layers to replace the IFE module, our proposed model 6 improves the PSNR from $25.4413 \mathrm{~dB}$ to $25.5489 \mathrm{~dB}$. This experiment demonstrates the vital role of IFE in our CVCnet.

8) Different Losses: We further study the influence of different losses on the performance of our CVCnet. As shown in Table VII, we used four different loss functions (i.e., SSIM, MSSIM, L1 and L2) to retrain our model. It can be seen from the table that the performance of the model trained with different loss functions varies greatly. Finally, we choose the L2 loss as our total loss for a better performance.

\section{CONCLUSION}

In this paper, we propose a cross view capture network for stereo image super-resolution by designing two innovative modules to explore rich feature representations across views. Specifically, we invent the CVB to capture both the global contextual and local feature embeddings between the left and right images in stereo pairs. Furthermore, SPM is presented to redistribute each pixel in the feature map according to the weight it occupies, and it makes the extraction of features more effective. The ablation study shows that our CVB and SPM can greatly improve the performance. The experimental results demonstrate that our CVCnet outperforms the state-of-the-art SR methods.

\section{ACKNOWLEDGMENT}

This work was supported in part by the Natural Science Foundation of China under Grant 62076255; in part by 
the Hunan Provincial Science and Technology Plan Project 2020SK2059; in part by the National Science Foundation of Hunan Province, China, under Grant 2019JJ20025 and Grant 2019JJ40406; in part by the National Social Science Fund of China (No. 20\&ZD120).

\section{REFERENCES}

[1] M. B. Islam, L. Wong, K. Low, and C. Wong, "Aesthetics-driven stereoscopic 3-d image recomposition with depth adaptation," IEEE Trans. Multimedia, vol. 20, no. 11, pp. 2964-2979, Mar. 2018.

[2] G. Sun, B. Shi, X. Chen, A. S. Krylov, and Y. Ding, "Learning local quality-aware structures of salient regions for stereoscopic images via deep neural networks," IEEE Trans. Multimedia, vol. 22, no. 11, pp. 2938-2949, Jan. 2020.

[3] D. Chen, L. Yuan, J. Liao, N. Yu, and G. Hua, "Stereoscopic neural style transfer," in Proc. IEEE Conf. Comput. Vision Pattern Recognit., 2018, pp. 6654-6663.

[4] C. Li, R. Cong, S. Kwong, J. Hou, H. Fu, G. Zhu, D. Zhang, and Q. Huang, "Asif-net: Attention steered interweave fusion network for rgb-d salient object detection," IEEE Trans. Cybern., vol. 51, no. 1, pp. 88-100, Feb. 2021.

[5] Z. Chen, R. Cong, Q. Xu, and Q. Huang, "Dpanet: Depth potentiality-aware gated attention network for rgb-d salient object detection," IEEE Trans. Image Process., to be published. DOI: 10.1109/TIP.2020.3028289.

[6] R. Cong, J. Lei, H. Fu, J. Hou, Q. Huang, and S. Kwong, "Going from rgb to rgbd saliency: A depth-guided transformation model," IEEE Trans. Cybern., vol. 50, no. 8, pp. 3627-3639, Aug. 2020.

[7] X. Yang, H. Mei, J. Zhang, K. Xu, B. Yin, Q. Zhang, and X. Wei, "Drfn: Deep recurrent fusion network for single-image super-resolution with large factors," IEEE Trans. Multimedia, vol. 21, no. 2, pp. 328-337, Aug. 2019.

[8] W. Yang, X. Zhang, Y. Tian, W. Wang, J. Xue, and Q. Liao, "Deep learning for single image super-resolution: A brief review," IEEE Trans. Multimedia, vol. 21, no. 12, pp. 3106-3121, May. 2019.

[9] C. Ma, Y. Rao, Y. Cheng, C. Chen, J. Lu, and J. Zhou, "Structurepreserving super resolution with gradient guidance," in Proc. IEEE Conf. Comput. Vision Pattern Recognit., 2020, pp. 7766-7775.

[10] Y. Guo, J. Chen, J. Wang, Q. Chen, J. Cao, Z. Deng, Y. Xu, and M. Tan, "Closed-loop matters: Dual regression networks for single image superresolution," in Proc. IEEE Conf. Comput. Vision Pattern Recognit., 2020, pp. 5406-5415.

[11] Z. He, Y. Cao, L. Du, B. Xu, J. Yang, Y. Cao, S. Tang, and Y. Zhuang, "Mrfn: Multi-receptive-field network for fast and accurate single image super-resolution," IEEE Trans. Multimedia, vol. 22, no. 4, pp. 10421054, Aug. 2020

[12] F. Yang, H. Yang, J. Fu, H. Lu, and B. Guo, "Learning texture transformer network for image super-resolution," in Proc. IEEE Conf. Comput. Vision Pattern Recognit., 2020, pp. 5791-5800.

[13] Z. Zhang, Z. Wang, Z. Lin, and H. Qi, "Image super-resolution by neural texture transfer," in Proc. IEEE Conf. Comput. Vision Pattern Recognit., 2019, pp. 7974-7983

[14] Y. Tian, Y. Zhang, Y. Fu, and C. Xu, "Tdan: Temporally-deformable alignment network for video super-resolution," in Proc. IEEE Conf. Comput. Vision Pattern Recognit., 2020, pp. 3360-3369.

[15] L. Wang, Y. Guo, L. Liu, Z. Lin, X. Deng, and W. An, "Deep video super-resolution using hr optical flow estimation," IEEE Trans. Image Process., vol. 29, pp. 4323-4336, Jan. 2020.

[16] D. S. Jeon, S. Baek, I. Choi, and M. H. Kim, "Enhancing the spatial resolution of stereo images using a parallax prior," in Proc. IEEE Conf. Comput. Vision Pattern Recognit., 2018, pp. 1721-1730.

[17] L. Wang, Y. Guo, Y. Wang, Z. Liang, Z. Lin, J. Yang, and W. An, "Parallax attention for unsupervised stereo correspondence learning," IEEE Trans. Pattern Anal. Mach. Intell., to be published. DOI: 10.1109/TPAMI.2020.3026899.

[18] W. Song, S. Choi, S. Jeong, and K. Sohn, "Stereoscopic image superresolution with stereo consistent feature," in Proc. AAAI Conf. Artif. Intell., 2020, pp. 12031-12038.

[19] C. Dong, C. C. Loy, K. He, and X. Tang, "Image super-resolution using deep convolutional networks," IEEE Trans. Pattern Anal. Mach. Intell., vol. 38, no. 2, pp. 295-307, Feb. 2016.

[20] J. Kim, J. K. Lee, and K. M. Lee, "Accurate image super-resolution using very deep convolutional networks," in Proc. IEEE Conf. Comput. Vision Pattern Recognit., 2016, pp. 1646-1654.
[21] K. He, X. Zhang, S. Ren, and J. Sun, "Deep residual learning for image recognition," in Proc. IEEE Conf. Comput. Vision Pattern Recognit., 2016, pp. 770-778.

[22] G. Huang, Z. Liu, L. Van Der Maaten, and K. Q. Weinberger, "Densely connected convolutional networks," in Proc. IEEE Conf. Comput. Vision Pattern Recognit., 2017, pp. 4700-4708.

[23] W. Lai, J. Huang, N. Ahuja, and M. Yang, "Deep laplacian pyramid networks for fast and accurate super-resolution," in Proc. IEEE Conf. Comput. Vision Pattern Recognit., 2017, pp. 624-632.

[24] M. Haris, G. Shakhnarovich, and N. Ukita, "Deep back-projection networks for super-resolution," in Proc. IEEE Conf. Comput. Vision Pattern Recognit., 2018, pp. 1664-1673.

[25] C. Guo, C. Li, J. Guo, R. Cong, H. Fu, and P. Han, "Hierarchical features driven residual learning for depth map super-resolution," IEEE Trans. Image Process., vol. 28, no. 5, pp. 2545-2557, Dec. 2019.

[26] Y. Mei, Y. Fan, Y. Zhou, L. Huang, T. S. Huang, and H. Shi, "Image super-resolution with cross-scale non-local attention and exhaustive self-exemplars mining," in Proc. IEEE Conf. Comput. Vision Pattern Recognit., 2020, pp. 5689-5698.

[27] J. Liu, W. Zhang, Y. Tang, J. Tang, and G. Wu, "Residual feature aggregation network for image super-resolution," in Proc. IEEE Conf. Comput. Vision Pattern Recognit., 2020, pp. 2356-2365.

[28] Y. Tai, J. Yang, and X. Liu, "Image super-resolution via deep recursive residual network," in Proc. IEEE Conf. Comput. Vision Pattern Recognit., 2017, pp. 3147-3155.

[29] B. Lim, S. Son, H. Kim, S. Nah, and K. M. Lee, "Enhanced deep residual networks for single image super-resolution," in Proc. IEEE Conf. Comput. Vis. Pattern Recognit. Workshops, 2017, pp. 1132-1140.

[30] N. Ahn, B. Kang, and K. A. Sohn, "Fast, accurate, and lightweight super-resolution with cascading residual network," in Proc. Eur. Conf. Comput. Vision, 2018, pp. 252-268.

[31] Z. Hui, X. Gao, Y. Yang, and X. Wang, "Lightweight image superresolution with information multi-distillation network," in Proc. ACM Int. Conf. Multimed., 2019, pp. 2024-2032.

[32] H. Zheng, M. Ji, L. Han, Z. Xu, H. Wang, Y. Liu, and L. Fang, "Learning cross-scale correspondence and patch-based synthesis for referencebased super-resolution," in Proc. Br. Mach. Vis. Conf., 2017, pp. 138.1138.13 .

[33] H. Zheng, M. Ji, H. Wang, Y. Liu, and L. Fang, "Crossnet: An end-to-end reference-based super resolution network using cross-scale warping," in Proc. Eur. Conf. Comput. Vision, 2018, pp. 87-104.

[34] A. Kappeler, S. Yoo, Q. Dai, and A. K. Katsaggelos, "Video superresolution with convolutional neural networks," IEEE Trans. Comput. Imaging, vol. 2, no. 2, pp. 109-122, Jun. 2016.

[35] J. Caballero, C. Ledig, A. Aitken, A. Acosta, J. Totz, Z. Wang, and W. Shi, "Real-time video super-resolution with spatio-temporal networks and motion compensation," in Proc. IEEE Conf. Comput. Vision Pattern Recognit., 2017, pp. 2848-2857.

[36] M. S. M. Sajjadi, R. Vemulapalli, and M. Brown, "Frame-recurrent video super-resolution," in Proc. IEEE Conf. Comput. Vision Pattern Recognit., 2018, pp. 6626-6634.

[37] Y. Jo, S. W. Oh, J. Kang, and S. J. Kim, "Deep video super-resolution network using dynamic upsampling filters without explicit motion compensation," in Proc. IEEE Conf. Comput. Vision Pattern Recognit., 2018, pp. 3224-3232.

[38] D. S. Jeon, S. Baek, I. Choi, and M. H. Kim, "Enhancing the spatial resolution of stereo images using a parallax prior," in Proc. IEEE Conf. Comput. Vision Pattern Recognit., 2018, pp. 1721-1730.

[39] B. Yan, C. Ma, B. Bare, W. Tan, and S. Hoi, "Disparity-aware domain adaptation in stereo image restoration," in Proc. IEEE Conf. Comput. Vision Pattern Recognit., 2020, pp. 13 176-13 184.

[40] W. Shi, J. Caballero, F. Huszar, J. Totz, A. P. Aitken, R. Bishop, D. Rueckert, and Z. Wang, "Real-time single image and video superresolution using an efficient sub-pixel convolutional neural network," in Proc. IEEE Conf. Comput. Vision Pattern Recognit., 2016, pp. 18741883.

[41] X. Wang, R. Girshick, A. Gupta, and K. He, "Non-local neural networks," in Proc. IEEE Conf. Comput. Vision Pattern Recognit., 2018, pp. $7794-7803$

[42] Z. Zhu, M. Xu, S. Bai, T. Huang, and X. Bai, "Asymmetric non-local neural networks for semantic segmentation," in Proc. IEEE Int. Conf. Comput. Vis., 2019, pp. 593-602.

[43] Y. Cao, J. Xu, S. Lin, F. Wei, and H. Hu, "Gcnet: Non-local networks meet squeeze-excitation networks and beyond," in Proc. IEEE Int. Conf. Comput. Vis. Workshops, 2019, pp. 1971-1980. 
[44] Z. Li, J. Yang, Z. Liu, X. Yang, G. Jeon, and W. Wu, "Feedback network for image super-resolution," in Proc. IEEE Conf. Comput. Vis. Pattern Recognit., 2019, pp. 3862-3871.

[45] C. Chen, C. Qing, X. Xu, and P. Dickinson, "Cross parallax attention network for stereo image super-resolution," IEEE Trans. Multimedia, to be published. DOI: 10.1109/TMM.2021.3050092.

[46] Y. Guo, J. Chen, J. Wang, Q. Chen, J. Cao, Z. Deng, Y. Xu, and M. Tan, "Closed-loop matters: Dual regression networks for single image superresolution," in Proc. IEEE Conf. Comput. Vis. Pattern Recognit., 2020, pp. 5406-5415.

[47] D. Scharstein, H. Hirschmüller, Y. Kitajima, G. Krathwohl, N. Nešić, $\mathrm{X}$. Wang, and P. Westling, "High-resolution stereo datasets with subpixel-accurate ground truth," in Proc. German Conf. Pattern Recognit., 2014, pp. 31-42.

[48] A. Geiger, P. Lenz, and R. Urtasun, "Are we ready for autonomous driving? the kitti vision benchmark suite," in Proc. IEEE Conf. Comput. Vision Pattern Recognit., 2012, pp. 3354-3361.

[49] M. Menze and A. Geiger, "Object scene flow for autonomous vehicles," in Proc. IEEE Conf. Comput. Vision Pattern Recognit., 2015, pp. 30613070.

[50] Q. H. Thu and M. Ghanbari, "Scope of validity of psnr in image/video quality assessment," Electron. Lett., vol. 44, no. 13, pp. 800-801, Jun. 2008.

[51] Z. Wang, A. C. Bovik, H. R. Sheikh, and E. P. Simoncelli, "Image quality assessment: from error visibility to structural similarity," IEEE Trans. Image Process., vol. 13, no. 4, pp. 600-612, Apr. 2004.

[52] Y. Zhang, K. Li, K. Li, L. Wang, B. Zhong, and Y. Fu, "Image superresolution using very deep residual channel attention networks," in Proc. Eur. Conf. Comput. Vision, 2018, pp. 286-301.

[53] T. Dai, J. Cai, Y. Zhang, S. Xia, and L. Zhang, "Second-order attention network for single image super-resolution," in Proc. IEEE Conf. Comput. Vision Pattern Recognit., 2019, pp. 11 065-11074.

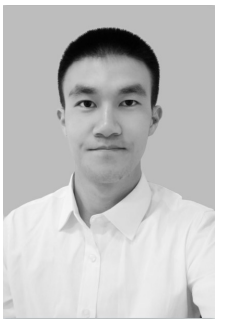

Xiangyuan Zhu received the B.E. degree in automation from Xiangtan University, Xiangtan, China, in 2017, and the M.E. degree in Control Science and Engineering from Central South University, Changsha, China, in 2020. He is currently pursuing the Ph.D. degree with the school of computer science and engineering with the Central South University. His research interests include low-level vision and deep learning.

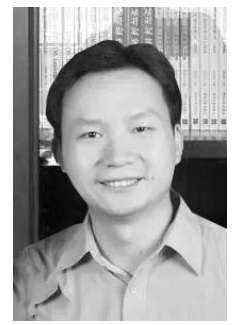

Kehua Guo received the Ph.D. degree in Computer Science and Technology from Nanjing University of Science and Technology, Nanjing, China, in 2008 $\mathrm{He}$ is currently a professor with Central South University, Changsha, China. He has served as a Guest Editor, Workshop Chair, Publicity Chair, Technical Program Committee Member, and Reviewer of international journals/conference proceedings. His research interests include social computing, ubiquitous computing, big data and image retrieval.

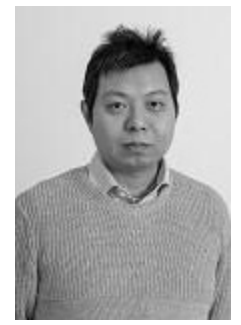

Hui Fang received the B.S. degree from the University of Science and Technology, Beijing, China, in 2000 and the Ph.D. degree from the University of Bradford, U.K., in 2006. He is currently with the Computer Science Department, Loughborough University (LU). Before joined LU, he has carried out research in several world-leading universities, such as University of Oxford and Swansea University. His research interests include computer vision, image/video processing, pattern recognition, machine learning, data mining, scientific visualization, visual analytics, and artificial intelligence. Recently, he was awarded several grants as PI and co-PI, including Innovate UK funded "An agent based modelling solution for reliable decision making in crisis and market turmoil in consumer retail", EPSRC funded "RAMP VIS: Making Visual Analytics an Integral Part of the Technological Infrastructure for Combating COVID-19", and NIHR funded "Computer vision to automatically monitor urine output". During his career, he has published more than 60 journal and conference papers.

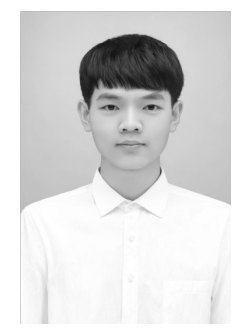

Liang Chen received his B.S. degree from Jiangxi University of Science and Technology in 2020 . He is currently a graduate student in the School of Computer Science and Engineering, Central South University, China. His research interests include image processing and big data.

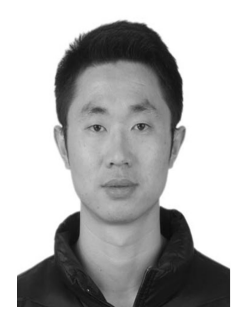

Sheng Ren received the M.S degree in Software Engineering in 2010 from Central South University, Changsha, China, where he is currently working toward the Ph.D. degree in computer science and engineering. His research interests include medical big data, image, and video super-resolution.

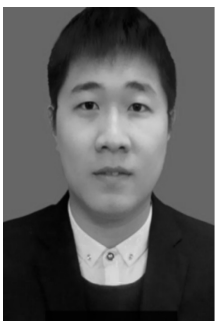

Bin Hu received a master's degree in Control Engineering from Central South University in 2019. He is currently a Ph.D. student in the School of Computer Science and Engineering, Central South University, Changsha, China. His research interests include video analysis and understanding, graph convolution networks and abnormal behavior recognition. 